\title{
Protée
}

\section{La fleur et le scarabée}

\section{Shawn Huffman}

Volume 27, numéro 1, 1999

\section{La Mort de Molière et des autres}

URI : https://id.erudit.org/iderudit/030543ar

DOI : https://doi.org/10.7202/030543ar

Aller au sommaire du numéro

Éditeur(s)

Département des arts et lettres - Université du Québec à Chicoutimi

ISSN

0300-3523 (imprimé)

1708-2307 (numérique)

Découvrir la revue

\section{Citer cet article}

Huffman, S. (1999). La fleur et le scarabée. Protée, 27(1), 63-68.

https://doi.org/10.7202/030543ar

\section{Résumé de l'article}

Cet article décrit la tension entre deux représentations de la mort dans le film La Mort de Molière. À partir des recherches de Greimas et de Fontanille sur la sémiotique tensive, il tâche d'identifier ces représentations, d'interroger la tension qu'elles produisent de même que les répercussions somatiques qui en découlent. d'utilisation que vous pouvez consulter en ligne.

https://apropos.erudit.org/fr/usagers/politique-dutilisation/ 


\title{
LA FLEUR ET LE SCARABÉE
}
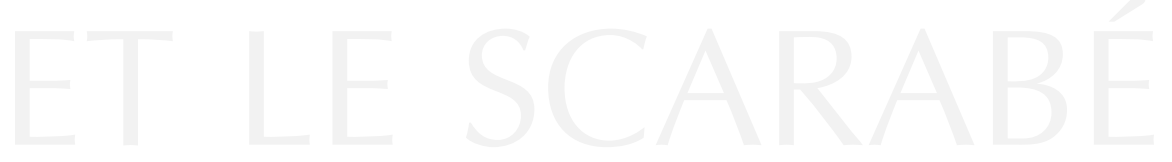

SHAWN HUFFMAN

\author{
Les écrivains \\ font dériver les morts. \\ Isolé, \\ le tumulus donnera sa poupe. \\ Nous sourcillerons alors, visibles, \\ tout à fait mimés. \\ Bertrand Laverdure, " Ponceau ».
}

"Je t'ai pourtant dit de ne pas revenir. Quand on est mort on est mort» (Wilson, 1994: section 8) ${ }^{1}$. Avec cette phrase, le narrateur de La Mort de Molière soulève la question centrale du film, à savoir celle de la permanence de la mort. Depuis au moins le mythe d'Orphée, le retour du trépassé ne cesse de fasciner les artistes, d'autant plus que si Orphée a réussi à convaincre la reine de l'Enfer de laisser partir Eurydice, c'est grâce à sa poésie et à sa musique. Toutefois, le trio artistique de Robert Wilson, Heiner Müller et Philip Glass ne cherchent pas à ressusciter Molière: «On ne peut pas écrire un poème sur Molière» (MM: 1). Ils proposent plutôt une interrogation des modalités de sa mort. D'une part, ils mettent en scène l'agonie du corps par les signes de son extinction: une plaie rouge sur le flanc, ou encore, une tache de sang sur un mouchoir. Ceux-ci prennent la forme d'une fleur en éclosion. D'autre part, les artistes font état d'un refus de la mort communiqué par le désir de conserver, voire de momifier le corps. Ce désir s'exprime sous le signe du scarabée, symbole égyptien de l'immortalité. Dans ce court article ${ }^{2}$, je me propose de décrire, dans un premier temps, la tension des modalités de la mort représentées par la fleur et par le scarabée. Dans un deuxième temps, me basant sur la sémiotique tensive, je tâcherai d'interroger cette tension de même que les répercussions somatiques qui en découlent.

\section{LA SÉMIOTIQUE TENSIVE}

Résultat du travail de Greimas et de Fontanille sur les passions, la sémiotique tensive constitue aujourd'hui un champ d'investigation des plus prometteurs en 
sémiotique. La littérature, les arts visuels, le théâtre et le cinéma ouvrent des horizons de recherche d'où l'on voit émerger une théorie de la signification qui permet de tenir compte non seulement de l'activité perceptivo-énonciative dans le discours, mais aussi de la dimension somatique et émotionnelle du sujet. Cet appel à un corps sensible dans le monde (Fontanille, 1995: 6) rejoint les recherches de Ouellet sur la sémiotique perceptive (1992) et signale une adaptation de la phénoménologie husserlienne et merleaupontienne à l'épistémologie sémiotique (Ouellet, 1996). D'autres chercheurs ont déjà montré l'importance de cette rencontre avec la phénoménologie par rapport à des concepts, comme le sujet percevant (Fontanille, 1995) ou l'intersubjectivité (Couégnas, 1998). Ces concepts ont le mérite de mettre en évidence l'émergence du sens en rapport avec un champ perceptif. Qu'en est-il cependant de la disparition ou de la diminution du champ perceptif, ou encore de l'extinction du sujet percevant ${ }^{3}$ ? C'est précisément cette question qui est posée par la vidéo de Robert Wilson.

Rappelons que dans La Sémiotique des passions, Greimas et Fontanille proposent deux visions complémentaires du monde: le monde continu et le monde discontinu. Ce dernier nous est familier puisque la différence constitue le mode de fonctionnement de la sémiotique depuis au moins Saussure. Le monde continu, lui, correspond à une existence sémiotique homogène qui précède la catégorisation. De là surgissent alors les préconditions de la signification. La pertinence de la vision continue dérive, en partie, de sa capacité à rendre compte des gradients et des fluctuations énergétiques qui participent à l'organisation précatégorielle de la signification. Il s'agit d'une sorte d'ombre qui accompagne le sens, une ombre de valeur liée à un sujet en voie d'émergence (Greimas et Fontanille, 1991: 26) ou en voie de disparition, comme nous le verrons.

Toujours selon Greimas et Fontanille, on peut décrire ces fluctuations énergétiques à l'intérieur de l'ombre de valeur par le biais de la valence. Celle-ci a lieu sur «un horizon de tensions inarticulées [où...] s'exercent $[. .$.$] les premières sommations du sujet$ opérateur, discrétisant et faisant apparaître les premières unités significatives» (1991: 15). Elle correspond à une espèce de polarisation énergétique qui se produit sur cet horizon. Pas encore un processus de catégorisation, elle se manifeste plutôt comme le premier "positionnement" de zones tensives qui seront par la suite organisées en unités de valeur et ce, en fonction du corps percevant. On tient compte de ce "positionnement" de deux manières: d'abord en fonction de sa tensivité, qui renvoie à une sorte de portrait du monde en voie d'émergence ou de disparition élaboré selon un axe de l'intensité et selon un axe de l'extensité 4 et, ensuite, en fonction de la phorie, qui correspond aux effets d'actes perceptivocognitifs qui dessinent un proto-sujet percevant ${ }^{5}$ sur l'horizon ontique. De plus, bien que les zones tensives précèdent l'articulation de sens, on ne doit pas penser la relation entre la valence et la valeur de façon linéaire ${ }^{6}$. Telle l'ombre qui «se colle» à son «objet», les zones tensives sont toujours présentes et risquent de se manifester en tout temps. Tournons-nous maintenant vers l'espace-temps tensif de La Mort de Molière.

\section{LA FLEUR}

«Galilée observe les étoiles» (MM: 1); ce qu'il y découvre bouleverse la cosmogonie de son époque et l'inscrit en rupture avec ses pairs. De même, dans La Mort de Molière, Wilson rompt avec une autre cosmogonie, celle selon laquelle «la France est une seule famille» (MM: 1$)$ dont Molière serait le pèredramaturge. Ce n'est qu'en décrochant le portrait de Molière - la vision muséologique de l'homme - que l'on peut remettre en question l'icône que l'on a substituée au corps. Dans le cas de Galilée comme dans celui de Wilson, c'est le refus de corroborer une version consacrée de l'univers qui ouvre, dans le discours, une brèche permettant d'entrevoir d'autres éventualités.

Galilée observe une comète, un corps céleste. Wilson, lui aussi, observe un corps, celui de Molière 
agonisant. Lors de ce spectacle de la mort, le narrateur fait état du déclin physique du dramaturge; il décrit en détail, par exemple, les convulsions et les malaises qui précèdent son extinction.

La sémiotique tensive permet de concevoir le corps sensible dans le monde. Le film de Wilson met en évidence ce corps, de même que la fracture émotionnelle occasionnée par l'irruption somatique dans le discours. Dans La Sémiotique des passions, Greimas et Fontanille décrivent l'émotion - la dimension thymique selon la terminologie consacrée - comme une doublure qui se rattache au discours. Or, un événement bouleversant ou passionnel fissure le discours et laisse entrevoir cette doublure qui correspond à un espace-temps tensif, c'està-dire à une zone d'énergies précatégorielle. Dans le film, la séquence de la commedia dell'arte révèle cette doublure passionnelle.

Si on considère la gestualité, par exemple, la position des mains de Molière avant et après la séquence correspond à celle des mains des personnages de la commedia dell'arte. Loin d'être un simple détail, ce lien est soigneusement souligné par l'emploi d'un gros plan qui met sous la loupe, pour ainsi dire, le débrayage du corps charnel et l'émergence concomitante d'une zone tensive. Les personnages sont des projections imaginaires d'un homme mourant - des projections passionnelles, diraient Greimas et Fontanille - et leur éloquence non langagière en dit long sur l'investissement somato-émotionnel du discours. De plus, puisqu'il s'agit d'une projection imaginée par le personnage de Molière, l'esthésie pratiquée est de nature réflexive, car elle pointe la relation entre le sujet et son propre corps. Dans La Sémiotique du visible, Fontanille fait appel à la notion de phorie pour rendre compte de l'activité corporelle dans de telles circonstances: [...] la phorie serait l'effet [...] sur le corps propre du sujet percevant, des variations de rapports de force dans l'espace tensif où il est plongé; elle permet au corps propre de défendre son intégrité au sein des forces qu'il ressent. (1995: 7)

La séquence de la commedia dell'arte renvoie alors très exactement à l'homme de théâtre même, à celui qui a vécu par le théâtre et qui se meurt avec lui. La doublure émotionnelle de Molière ne contient rien de moins que la mise en scène de son propre corps. Cette hypothèse d'une esthésie réflexive mettant en vedette le corps du personnage est étayée par le personnage du médecin.

La médecine, selon le narrateur du film, est «une des grandes erreurs de l'humanité» (MM: 4), surtout parce qu'elle intervient dans l'évolution naturelle du corps, évolution qui comprend nécessairement le décès. Molière, par exemple, reçoit des comprimés pour stopper les ravages de sa maladie et prolonger sa vie. Malgré cela, la mort continue de grandir en lui et, comme le garçon dans «Un Médecin de campagne» de Kafka, qui "péri[t] de cette fleur dans [s]on flanc" $(\mathrm{MM}: 4)^{7}$, Molière, lui aussi, fait éclore une fleur, celle dépeinte par les taches de sang sur le tissu du mouchoir blanc dans lequel il crache. C'est un indice de sa fragilité, signe de la mortalité de son corps. «Où dort mon renard en hiver?» demande le narrateur, «Où se terre mon serpent?» (MM: 7). Ces questions sont capitales puisqu'elles nous permettent d'identifier l'horizon tensif dans lequel le corps s'estompe. L'hiver comme la mort participent, notons-le, d'une aspectualité commune, à savoir la terminativité. Cela se manifeste aussi sur le plan émotionnel quand le jeune Molière lit à haute voix un passage du De rerum natura, où Lucrèce décrit les premières émissions nocturnes chez l'adolescent comme étant à la fois le zénith et le nadir de la perception sensuelle et amoureuse:

[...] son muet désir lui présage la volupté. Telle est pour nous

Vénus, telle est la réalité qui se nomme amour; voilà la source de la donce rosée qui s'insinue goutte à goutte dans nos coeurs et qui plus tard nous glace de souci. (1964: 144-145)

Cette aspectualité terminative généralisée 8 accompagne la valeur proprement dite; elle existe en deçà des contenus sémantiques articulés dans le discours et donne lieu à une doublure valencielle régie par la fin, par la mort. Or, contrairement à certains artistes chez qui le sursaut, le transport ou encore le frémissement témoignent d'une régression temporaire 
vers un champ de valence ${ }^{9}$, Wilson dépeint un retour en permanence. Le corps signifiant et signifié est réabsorbé dans un monde continu, le sujet devenant ainsi l'ombre de lui-même. Dans le film, cette régression s'opère très exactement à travers une autre projection passionnelle par laquelle le sujet Molière vit ses dernières minutes dans le rôle de Dom Juan qui descend aux enfers. Encore une fois, on remarquera que la doublure qui accompagne le discours est de facture théâtrale, car la «mort» de Molière s'exprime par le biais du théâtre. Le choix du théâtre pour communiquer le décès de Molière, ainsi que les autres manifestations phoriques exprimées dans le film inscrivent sa mort sous le signe de la fleur, qui est de l'ordre de la fragilité et de la fugacité. Il existe, cependant, une autre modalité de la mort dans le film, celle représentée par le scarabée.

\section{LE SCARABÉE}

La médecine est décrite comme une nouvelle religion, mais qui, comme d'autres religions, proclame le triomphe sur la mort, le corps ressuscité et la vie éternelle. Entouré de religieuses qui chuchotent des prières, le personnage du médecin célèbre ainsi une communion, son calice, un pot de chambre et son vin, l'urine de ses patients (MM: 4). Le médecin lutte contre la pourriture. Son rôle est de préserver le corps, de le protéger contre les éléments qui entraîneraient son anéantissement. Le texte de Kafka cité dans le film confirme cette vocation: " $\mathrm{Me}$ sauveras-tu?" souffle entre deux sanglots le garçon complètement hypnotisé par la vie qui grouille dans sa blessure» (MM: 4). Mais la mort est un opposant de taille et la médecine, comme nous le rappelle le texte de Plutarque cité dans le film (MM: 2bis), encaisse des défaites spectaculaires malgré l'instinct de survie qui incite l'être humain à placer sa foi en elle. "Tels sont les gens de ma contrée», observe le médecin citant Kafka:

Ils exigent toujours l'impossible du médecin. Ils ont perdu l'ancienne foi; le prêtre reste chez lui et transforme en charpie les ornements sacerdotaux l'un après l'autre; mais le médecin doit tout faire de sa main légère de chirurgien. (MM: 4)
Mais de quel corps s'agit-il au juste dans ce film? Quel est l'objet de la médecine?

Par le biais de l'intertextualité, Wilson fait revivre le texte de Kafka, c'est-à-dire le corpus textuel. De cette manière, la "nouvelle religion" trafique le souffle contre le signe: indice, icône, symbole. Le médecin est celui qui effectue cette transsubstantiation, mais à l'envers, puisque la chair se transforme en signe. Molière, bien entendu, subit cette transformation. Voyons comment.

Madeleine, la défunte amante du dramaturge, et Armande, sa femme et la fille de Madeleine, se présentent au chevet de Molière. Pendant qu'elles l'observent, on récite un texte qui est une adaptation des Femmes savantes. Par conséquent, on n'est pas seulement en présence du corps de Molière, mais aussi de son corpus. Madeleine, ou plutôt le spectre de cette dernière, est tout de blanc vêtue. Elle revient de la mort pour apporter un message au dramaturge, mais ce n'est pas un message de mots qu'elle apporte. Justement, la parole se désintègre au fur et à mesure que la scène se déroule; le souffle se solidifie et cède la place au symbole. L'ancienne amante de Molière retire de sa bouche un scarabée, le plaçant sur la main de Molière. Molière (mais lequel?) ne mourra jamais.

L'insecte est noir, comme les textes carbonisés du dramaturge dans l'avant-dernière séquence. Le scarabée est un hiéroglyphe qui signifie très exactement l'autre corps qui rendra Molière immortel, le corps d'écriture conservant les signes de la vie et de la mort de l'auteur. Ce passage par le discours est même obligatoire - comme le décrit Linders, la recherche sur la mort de Molière a été menée dans le discours, soit fictif comme le roman de Boulgakov, soit "véridique», comme les écrits sur l'éducation jésuite du dramaturge (1996: 93-95). De cette façon, l'écriture correspond à un sépulcre qui dissimule la dépouille de Molière.

À l'entrée de la Comédie-Française, on peut observer un sarcophage de verre, à l'intérieur duquel se trouve le fauteuil dans lequel Molière a joué sa dernière pièce, Le Malade imaginaire. Cette image devient un leitmotiv dans le film, juxtaposée à la 
version du dramaturge en chair et en os. Plus qu'un symbole fétiche du corps trépassé, le fauteuil représente très concrètement la chair momifiée du dramaturge. La momification elle-même est un processus continu, comme le démontre la scène où l'on tâche d'appliquer une graisse au cuir du fauteuil afin de le préserver. Ou encore, quand le médecin passe derrière le sarcophage de verre et s'aperçoit que la porte est ouverte; il la referme et scelle de nouveau le tombeau de Molière. La cage transparente représente très exactement la poésie; c'est une cage en vers ${ }^{10}$. La poésie (au sens large) est donc ce qui préserve le corpus et ce qui permet de se représenter le corps.

Malgré ce qui semble être dans le film un parti pris pour le corps en chair et en os, plusieurs éléments suggèrent une tension ou un équilibre entre la mort articulée sous le signe de la fleur et celle exprimée par le symbole du scarabée. Comme dans le roman As I Lay Dying de Faulkner, la voix du trépassé nous rappelle le corps qui a cédé la place au symbole, sans pour autant s'effacer. Dans le film, cette tension provoque une confusion chez le narrateur:

Voulez-vous que je parle de moi Moi qui

De qui est-il question quand

Il est question de moi Qui est-ce moi

Ou encore Moi un drapeau un

Lambeau sanglant à la fenêtre Un flottement

Entre le néant et personne à condition qu'il y ait du vent (MM: 10)

Extrait «troué» du Paysage avec Argonautes de Müller, ce texte met en évidence une «synthèse du moi». Plus précisément, celui-ci appartient à la fois à l'espace tensif, à l'espace sémio-narratif et à l'espace discursif. Le corps phorique se rattache au discours et s'anime en lui. Il est la mémoire du souffle derrière le signe, premier et dernier appel de la vie par la mort.

\section{CONCLUSION}

Dans Sade, Fourrier, Loyola, Barthes répudie l'affirmation de la mort de l'auteur qu'il avait avancée déjà dans Le Bruissement de la langue. Il dit ne plus croire au remplacement de l'auteur par un pur construit (un ressenti?) lectoriel. En effet, comment l'auteur des Fragments d'un discours amoureux pouvait-il ne pas modifier une telle position, le corps perceptivoémotif étant inscrit à presque toutes les pages du célèbre essai? Dans La Mort de Molière, Wilson interroge ce corps par le biais de sa mort, soulignant par là même la fragilité et le caractère éphémère de l'existence humaine. Toutefois, le dramaturge américain ne se limite pas à une naïve affirmation de la vie inscrite en opposition avec le signe. Au contraire, un travail important sur le discours révèle que le corps et le signe sont inextricablement liés dans la mort, que la fleur et le scarabée vont de pair, pris dans une tension signifiante.

\section{NOTES}

1. Toute référence ultérieure à cette œuvre sera indiquée dans le texte par MM, suivi du numéro de la section.

2. Cet article s'insère dans le cadre d'un projet de recherche postdoctoral subventionné par le CRSH que je remercie de son soutien financier.

3. Une ébauche de cette problématique est présentée par Huffman, 1997.

4. L'explication de Couégnas est claire et concise: «[...] ces deux ordres de grandeurs de l'intensité, hérités de la linguistique hjelmslévienne, permettent la mesure des phénomènes survenant dans l'univers tensif [...]. Chaque esquisse pénétrant dans le champ de présence d'un sujet est assujettie à la corrélation de l'intense et de l'extense. Soit l'esquisse est perçue de façon dominante comme extense lorsqu'elle s'étend dans la durée et l'espace, et l'intensité est alors faible, soit la perception de l'esquisse tend à se concentrer et son intensité croît» (1997: 217).

5. L'imbrication du proto-sujet et de la valence est explicitée par Greimas et Fontanille; il s'agit d'un «sujet protensif indissolublement lié à une "ombre de valeur", se profilant sur l'écran de la "tensivité phorique" "(1991: 26).

6. Voir Fontanille et Zilberberg, 1996.

7. Ce texte est cité lors de la séquence du médecin.

8. Il faudrait aussi évoquer le langage. À ce sujet, notons que Molière agonisant ne parle plus, il jappe plutôt, communiquant par là même la fin de sa participation au langage.

9. Voir Greimas, De l'imperfection, 1987.

10. On joue beaucoup sur les homonymes de verre: le vers poétique, les vers qui grouillent au fond d'une plaie. 


\section{RÉFÉRENCES BIBLIOGRAPHIQUES}

COUÉGNAS, N. [1997]: "Travers du temps: champs perceptifs et temporalité chez Proust et Yourcenar", RS/SI, vol. 17, no 1-3, 211-231;

[1998]: «Intersubjectivité et tensitivité dans Tu ne t'aimes pas de Nathalie Sarraute", Protée, vol. 26, nº 1, 145-150.

FAUlKNER, W. [1985]: As I Lay Dying, New York, Vintage International. FONTANILlE , J. [1995] : Sémiotique du visible: des mondes de lumière, Paris, P.U.F.

FOnTANille , J. ET C. ZilberberG [1996]: "Valence/Valeur», Nouveaux Actes sémiotiques, no 46-47, 13-69.

Greimas, A.J. [1987]: De l'imperfection, Périgueux, Fanlac.

Greimas, A.J. et J. FOnTANILLE [1991] : Sémiotique des passions : des états de choses aux états d'âme, Paris, Seuil.

HufFman, S. [1997]: "Charting Affect Through Drift in Robert

Lepage's Tectonic Plates", RS/SI, vol. 17, no 1-3, 193-210.

LAVERdure, B. [1996] : Fruits, Montréal, Noroît.

LINDERS, J. [1996] : «Molière \pm Müller », Performance Research, vol. $1, \mathrm{n}^{\circ} 2,93-102$.
LUCRĖCE [1964]: De la nature, trad. Henri Clouard, Paris, GF-

Flammarion.

Marlowe, C. [1962] : The Plays of Christopher Marlowe, Leo Kirschbaum (s.l.n.d.), New York, Meridian Books.

OUELlET, P. [1992] : Voir et savoir: la perception des univers du discours, Montréal, Balzac;

[1996]: "Pour une sémiotique tensive: les gradients du sens ", Nouveaux Actes sémiotiques, no 46-47, 3-12.

RozIK, E. [1998]: «Non-Theatrical Space as Metaphor: Some Spectulations on H.G. an Installation by Hans Peter Kuhn and Robert Wilson", dans E. Hess-LütTICH et al, Signs E⿱ Space, Raum Ë Zeichen: An International Conference on the Semotics of Space and Culture in Amsterdam, Tübingen, Gunter Narr Verlag.

WiLson, R. [1994] : La Mort de Molière, Vidéo couleur HD, 47 min, Paris, La Sept/ARTE;

[1998]: «Une Vie dans le prisme du théâtre ", Mouvement : revue du spectacle vivant et des arts visuels [Paris] 1 (juin-juillet-août), 40-50. 